\title{
1-(3-C-Ethynyl-B-D-ribo-pentofuranosyl)cytosine (ECyd, TAS-106), a novel potent inhibitor of RNA polymerase, potentiates the cytotoxicity of CDDP in human cancer cells both in vitro and in vivo
}

\author{
HIROMI KAZUNO ${ }^{1}$, AKIO FUJIOKA ${ }^{1}$, MASAKAZU FUKUSHIMA ${ }^{2}$, \\ YUSUKE WATAYA $^{3}$, AKIRA MATSUDA ${ }^{4}$ and TAKUMA SASAKI ${ }^{5}$ \\ ${ }^{1}$ Hanno Research Center, Taiho Pharmaceutical Co., Ltd., 1-27, Misugidai, Hanno-city, Saitama 357-8527; \\ ${ }^{2}$ Tokushima Research Center, Taiho Pharmaceutical Co., Ltd., 224-2, Hiraishi-ebisuno, Kawauchi, Tokushima 771-0194; \\ ${ }^{3}$ Faculty of Pharmaceutical Sciences, Okayama University, 1-1-1 Tsushimanaka, Okayama 700-8530; \\ ${ }^{4}$ Graduate School of Pharmaceutical Sciences, Hokkaido University, Kita-12 Nishi-6, Kita-ku, Sapporo 060-0812; \\ ${ }^{5}$ Pharmaceutical Science, Aichi-Gakuin University, 1-100, Kusumoto-cho, Chikusa-ku, Nagoya 464-8650, Japan
}

Received December 11, 2008; Accepted February 6, 2009

DOI: 10.3892/ijo_00000264

\begin{abstract}
C-Ethynyl-ß-D-ribo-pentofuranosyl)cytosine (ECyd, TAS-106) is a novel antitumor ribonucleoside that inhibits RNA polymerase. In the present study, we investigated the cellular and molecular interactions between TAS-106 and cisplatin (CDDP) in vitro using A549 human lung cancer cells and the in vivo antitumor effect of combined treatment using OCC-1 and LX-1 human tumor xenografts. The treatment effects were determined by evaluating cytotoxicity, the cell cycle distribution, apoptosis induction and the expression of checkpoint-associated proteins. In vitro, the combination of TAS-106 and CDDP synergistically inhibited the growth of A549 cells, as determined using isobologram analysis. TAS-106 potently inhibited the expression of Chk1 protein and the phosphorylation of Chk1 and Chk2. Moreover, based on the inhibition of checkpoint-associated protein, TAS-106 abrogated the CDDP-induced S- and $\mathrm{G}_{2} \mathrm{M}$ checkpoints and induced apoptosis in A549 cells. In vivo, TAS-106 alone showed antitumor activity; however, its combination with CDDP significantly enhanced the growth inhibition of OCC-1 and LX-1 tumors. Moreover, combination therapy with TAS-106 and CDDP in the OCC-1 xenograft model resulted in significant life-prolongation. These
\end{abstract}

Correspondence to: Dr Hiromi Kazuno, Hanno Research Center, Taiho Pharmaceutical Co., Ltd., 1-27, Misugidai, Hanno-city, Saitama 357-8527, Japan

E-mail: h-kazuno@taiho.co.jp

Key words: combination chemotherapy, 1-(3-C-ethynyl-ß-D-ribopentofuranosyl)cytosine, ECyd, TAS-106, cisplatin, apoptosis, $\mathrm{G}_{2} \mathrm{M}$ abrogation, Chk1, Chk2 findings provide a rationale for combination chemotherapy using TAS-106 and CDDP in clinical settings.

\section{Introduction}

1-(3-C-Ethynyl-ß-D-ribo-pentofuranosyl)cytosine (3'-ethynylcytidine, ECyd, TAS-106) is a new antitumor cytidine analogue possessing potent cytotoxic and antitumor activities in preclinical therapeutic models (1-4) and phase I/II clinical trials examining the use of TAS-106 as monotherapy and in combination with other cytotoxic drugs have recently begun. Previous studies have revealed that the cytotoxic effects of TAS-106 are mainly related to the inhibition of RNA biosynthesis $(5,6)$. TAS-106 is converted into 3'-ethynylcytidine-5'monophosphate (ECMP) by uridine-cytidine kinase (UCK, EC 2.7.1.48) (7) and this product is subsequently phosphorylated to 3'-ethynylcytidine-5'-diphosphate (ECDP) and finally to 3'-ethynylcytidine-5'-triphosphate (ECTP). Moreover, RNA polymerase was inhibited competitively by ECTP in the presence of isolated nuclei from FM3A mouse tumor cells. The Ki value of ECTP was $20 \mathrm{nM}$, while the apparent $\mathrm{Km}$ value of RNA polymerase for CTP was $8 \mu \mathrm{M}$ (8). ECTP was found to be a major intracellular metabolite that accumulates abundantly in cells exposed to TAS-106 because of its slow elimination from these cells.

Cis-diamminedichloroplatinum (II) (CDDP) is one of the most effective antitumor agents available for the treatment of testicular, ovarian, bladder, head and neck, small cell lung and cervical cancer. The main mechanism of CDDP cytotoxicity is likely exerted through covalent binding to DNA. The binding of CDDP induces both interstrand and intrastrand cross-links in DNA structure, inhibiting replication and transcription processes. However, the presence of a DNA repair system means that these structural lesions are only transient. In many instances, a high level of DNA repair may significantly abolish the antitumor activity of CDDP (9). When CDDP damages 
DNA, cell cycle progression is temporary blocked at $\mathrm{G}_{2}$ phase to facilitate DNA repair. If the DNA lesions are not repaired, however, the cells may enter an apoptotic pathway (10).

In general, combination chemotherapy with other agents that have different mechanisms of activity is performed in the hope of attaining a high antitumor efficacy. For this reason, accumulating rationale for combination chemotherapy is a useful endeavor for designing effective combination regimens with other anticancer agent(s).

The purpose of this study was to clarify whether the combination of TAS-106 and CDDP would produce a more potent antitumor effect. Indeed, the combination of both compounds was very effective, not only in an in vitro system but also in an experimental xenograft model in vivo.

\section{Materials and methods}

Chemicals. TAS-106 was synthesized at Taiho Pharmaceutical Co., Ltd. (Tokyo, Japan). Cis-diamminedichloroplatinum(II) (CDDP, Briplatin ${ }^{\circledR}$ injection) was obtained from BristolMyers Squibb Co., Ltd. All other chemicals were of analytical grade and were purchased from commercial sources.

Cell lines and cell culture. Human lung cancer A549 cells were obtained from the American Type Culture Collection (Rockville, MD). A549 cells were maintained in Dulbecco's modified Eagle's medium (DMEM) supplemented with $10 \%$ heat-inactivated fetal bovine serum (FBS; ICN Biomedicals, Inc., Aurora, $\mathrm{OH}$ ) at $37^{\circ} \mathrm{C}$ in $5 \% \mathrm{CO}_{2}$.

Tumor xenografts. LX-1 (human lung cancer) and OCC-1 (human oral cancer) xenografts were obtained from the Cancer Chemotherapy Center, Japanese Foundation for Cancer Research and the Central Institute for Experimental Animals, respectively.

Analysis of the effects of combination chemotherapy in vitro. For simultaneous exposure to TAS-106 and CDDP, A549 cells were treated with both drugs for $24 \mathrm{~h}$. After treatment, the cells were washed three times with culture medium and subsequently incubated for 2 days. For sequential exposure to TAS-106 and CDDP, A549 cells were treated with TAS-106 (or CDDP) for $24 \mathrm{~h}$ and then washed three times with culture medium. Subsequently, the cells were treated with CDDP (or TAS-106) for $24 \mathrm{~h}$. After treatment, the cells were washed three times with culture medium and were subsequently incubated for 1 day.

Viable cell growth was evaluated using a tetrazolium saltbased (MTT, $\mathrm{C}_{18} \mathrm{H}_{16} \mathrm{~N}_{5} \mathrm{SBr}$ ) semi-automated colorimetric assay (11). The measurement of MTT-formazan product, absorbed at $540 \mathrm{~nm}$, was performed using a Model 3550 microplate reader (Bio-Rad Laboratories, Hercules, CA).

Dose response interactions between TAS-106 and CDDP at the point of $\mathrm{IC}_{50}$ were evaluated using the isobologram method of Steel and Peckham $(12,13)$.

Cell cycle analysis. A549 cells were treated with TAS-106, CDDP or both drugs using various schedules. The cells were then collected and stained with propidium iodide (PI) using the Cycle Test Plus DNA Reagent kit, according to the protocol provided by the manufacturer (Becton-Dickinson Immunocytometry Systems, San Jose, CA). Flow cytometric analysis was performed using a FACSCalibur flow cytometer (Becton-Dickinson, Franklin Lake, $\mathrm{NJ}$ ) and the data were subsequently analyzed using CellQuest ${ }^{\mathrm{TM}}$ software (BectonDickinson).

DNA fragmentation (TUNEL assay). DNA fragmentation was analyzed using the APO-BRDU ${ }^{\mathrm{TM}}$ assay (14), according to the protocol provided by the manufacturer (PharMingen, San Diego, CA). Briefly, A549 cells were treated with TAS-106, CDDP or both drugs for $72 \mathrm{~h}$ and then collected. Subsequently, the cells were stained with FITC-labeled deoxyuridine triphosphate (dUTP) using terminal deoxynucleotidyl transferase (TdT). The percentage of apoptotic cells in the tested samples was estimated using a FACSCalibur flow cytometer and subsequently analyzed using CellQuest software.

SDS-PAGE and Western blotting. A549 cells were treated with TAS-106, CDDP or both drugs for $24 \mathrm{~h}$ and then collected in SDS-PAGE sample buffer containing protease and phosphatase inhibitors after washing the cells in PBS. Cell lysates at the same protein concentration were separated on a $10 \%$ sodium dodecyl sulfate-polyacrylamide gel using electrophoresis and were transferred to a polyvinylidene difluoride membrane. The membranes were probed with anti-Chk1 (G-4) antibody (Santa Cruz Biotechnology, Inc., Santa Cruz, CA) diluted 1:500, anti-Chk2 (H-300) antibody (Santa Cruz Biotechnology, Inc.) diluted 1:500, anti-phosphoChk1 (Ser345) antibody (Cell Signaling Technology, Inc., Beverly, MA) diluted 1:1000, anti-phospho-Chk2 (Ser19) antibody (Cell Signaling Technology, Inc.) diluted 1:1000, and anti-ß-actin (clone AC-74) antibody (SIGMA) diluted 1:5000 overnight at $4^{\circ} \mathrm{C}$. The specific protein signals were detected using enhanced chemiluminescence by the appropriate secondary horseradish peroxidase-conjugated antibodies and the SuperSignal ${ }^{\circledR}$ West Dura Extended Duration Substrate (Pierce).

Antitumor effect and life-prolonging effect of combination therapy. LX-1 and OCC-1 tumor fragments $\sim 2 \mathrm{~mm}^{3}$ in size were transplanted s.c. into male F344/N Jcl-rnu nude rats (CLEA Japan, Inc., Tokyo). After reaching a tumor volume of $\sim 250 \mathrm{~mm}^{3}$, the rats were randomly assigned to control group and drug treated groups, each consisting of six animals (day 0 ) in the case of LX-1 human tumor xenografts. In the case of the study on OCC-1 tumor xenografts, 11 rats were assigned to each experimental group and then tumor fragments were transplanted into rats (day 0). Drugs were administered intravenously at a volume of $1 \mathrm{ml} / 100 \mathrm{~g}$ body weight starting the next day (day 1). TAS-106 was intravenously administered once a week for 2 weeks on LX-1, and intermittently (three times a week) for 2 weeks on OCC-1, respectively. CDDP was intravenously administered on Day 1. Tumor volume was measured using microcalipers two times weekly. The tumor growth inhibition rate (IR, \%) was calculated as the ratio of the mean tumor volume of the tumors treated to that of the control tumors (T/C) according to the formula: (1-T/C) x 100 . A life prolonging effect, indicated as an increase in life-span 

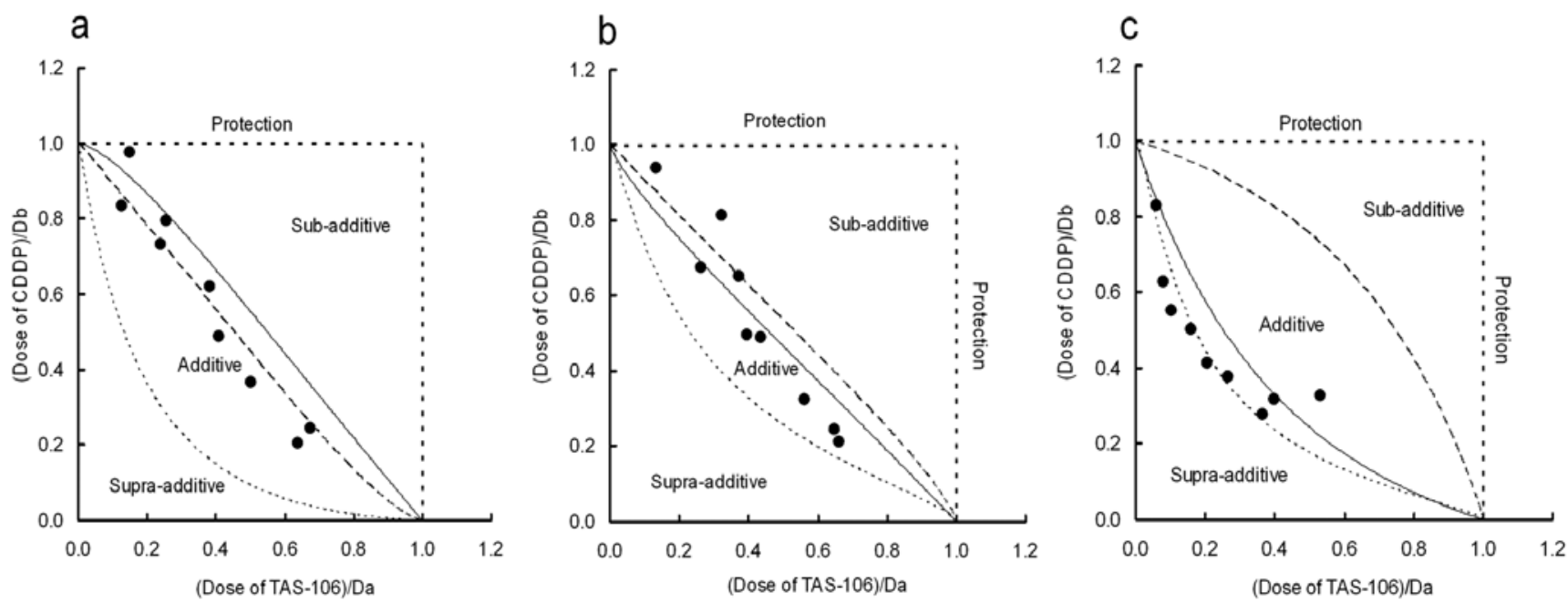

Figure 1. The combined effect of TAS-106 with CDDP was analyzed using the isobologram analysis according to Steel and Peckham at the point of $\mathrm{IC}_{50}$ values for A549 cells. (a) Simultaneous exposure to TAS-106 (24 h) and CDDP (24 h). (b) Sequential exposure to TAS-106 (24 h) followed by CDDP (24 h).

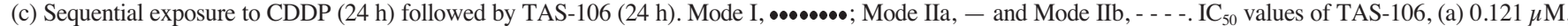
(b) $0.137 \mu \mathrm{M}$ and (c) $1.03 \mu \mathrm{M}$. IC I0 $_{0}$ values of CDDP, (a) $4.51 \mu \mathrm{M}$, (b) $14.0 \mu \mathrm{M}$ and (c) $4.11 \mu \mathrm{M}$.

(ILS, \%), was determined using the following formula: ILS $(\%)=($ mean survival time of treatment group/mean survival time of control group-1) x 100. All animal experiments were carried out in accordance with the Guidelines for the Welfare of Animals in Experimental Neoplasma.

Statistical analysis. The statistical analysis was performed using the Student's t-test and the Wilcoxon test for the animal experiments. A linear regression model using JMP ver.7 software (SAS Institute Inc.) was used to evaluate the results of the DNA fragmentation assay. In all of the statistical evaluations, $\mathrm{P}<0.05$ was considered to denote a significant difference.

\section{Results}

Combination effect of TAS-106 and CDDP in vitro. A549 cells were exposed to TAS-106 and CDDP for $24 \mathrm{~h}$ according to various schedules, e.g., simultaneous or sequential exposure. Isobolograms at the $\mathrm{IC}_{50}$ level were generated using dose response curves for the combinations. For simultaneous exposure (Fig. 1a) and sequential exposure to TAS-106 followed by CDDP (Fig. 1b), the data points for the combination fell within the envelope of additivity, suggesting the presence of additive effects. However, prior exposure of the cells to CDDP and then to TAS-106 resulted in a strong interaction, suggesting an almost supra-additive effect (Fig. 1c).

Variation in cell cycle according to exposure schedules. We compared the cell cycle profiles of cells subjected to various exposure schedules using the $\mathrm{IC}_{50}$ value of each drug after $72 \mathrm{~h}$ of exposure. For the monotherapy investigations, the cells were treated with TAS-106 or CDDP for $24 \mathrm{~h}$ and subsequently washed three times with culture medium and cultured for $24 \mathrm{~h}$ with drug-free medium. TAS-106 treatment of the A549 cells caused arrest at the G1-S boundary in addition to a significant reduction of cells in S-phase. The cells treated with CDDP maintained a significant increase in $\mathrm{S}$-phase or $\mathrm{G}_{2} \mathrm{M}$-phase. On the other hand, when we investigated concomitant treatment, the cell cycle analyses were performed after simultaneous or sequential exposures using A549 cells collected at 24 and 48 h, respectively. Significantly different perturbations in the cell cycle were also observed after these treatments. These results are shown in Fig. 2.

Augmentation of apoptosis by the combination of TAS-106 and $C D D P$. The extent of apoptosis was determined based on DNA fragmentation (TUNEL assay). To show the apoptotic events and the associated molecular changes more clearly, we used the drugs at higher concentrations. These results are shown in Fig. 3. The rate of apoptotic events (\%) after $72 \mathrm{~h}$ of exposure to $0.1 \mu \mathrm{M}$ TAS-106, $0.25 \mu \mathrm{M}$ TAS-106 and $12.5 \mu \mathrm{M}$ CDDP alone were $0.67,5.46$ and 0.56 , respectively. The combination of these agents at the same concentrations resulted in a dramatic increase in the number of cells undergoing apoptosis. The TUNEL positive rates for combined treatment with either $0.1 \mu \mathrm{M}$ or $0.25 \mu \mathrm{M}$ TAS-106 and $12.5 \mu \mathrm{M}$ CDDP were 12.4 and $55.6 \%$, respectively. These results suggest that the combination of TAS-106 and CDDP exert a synergistic augmentation of the cytotoxic effect $(\mathrm{p}<0.05)$. Such strong induction of apoptosis is expected to contribute significantly to the antitumor activity.

TAS-106 abrogates CDDP-induced cell cycle checkpoints. The changes observed in the cell cycle after combined treatment prompted us to study whether those effects resulted from an eventual imbalance in molecular events controlling cell cycle progression. The changes in the expression of the checkpoint-associated proteins were evaluated after 24-h exposure under the same conditions which were used in the experiments on the induction of the apoptotic events. Such approach might allow us to confirm an association between early molecular events and a process of cell death. The effects 

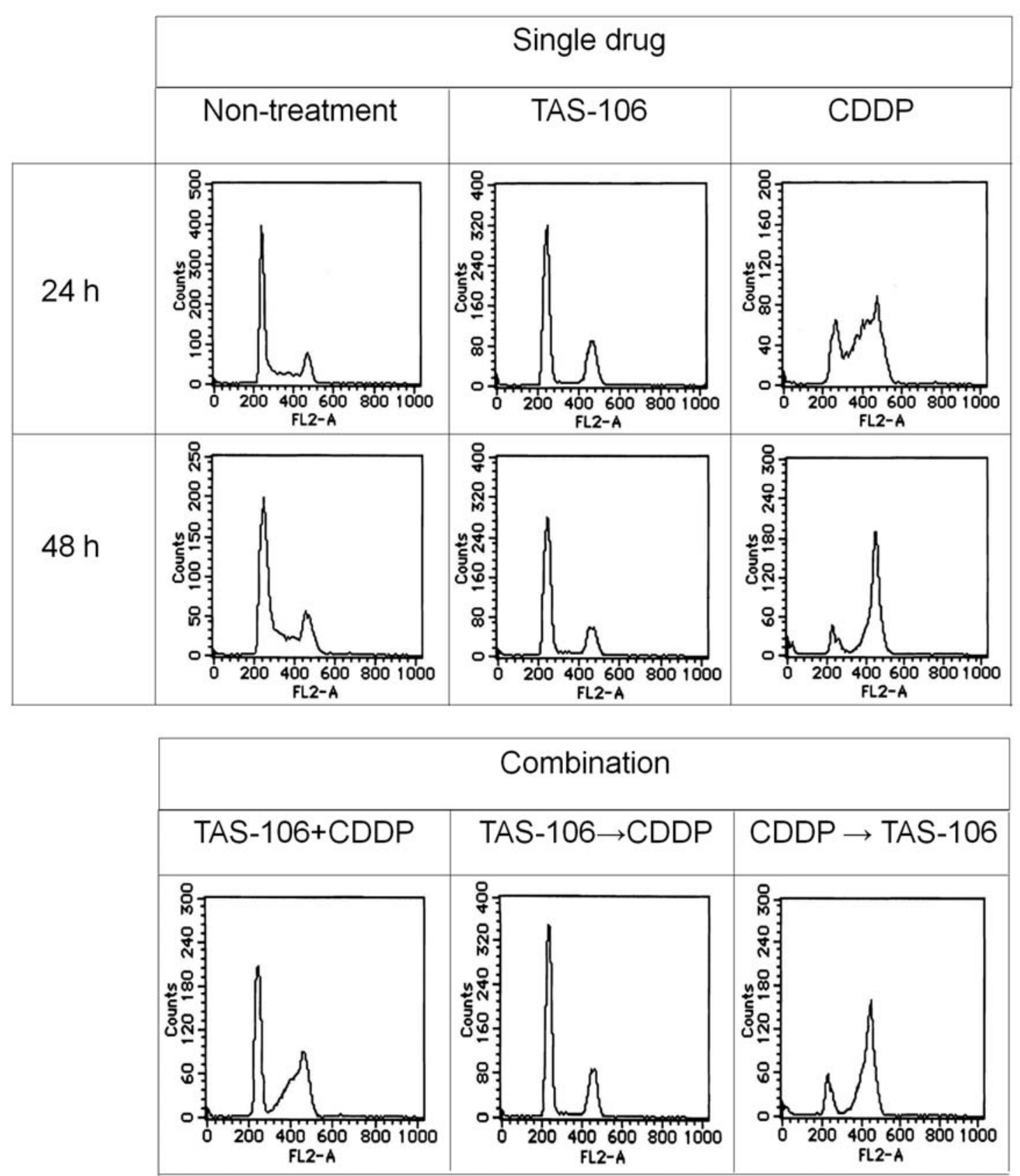

Figure 2. Variation in cell cycle according to exposure schedules. For the monotherapy, the cells were treated with TAS-106 or CDDP for $24 \mathrm{~h}$ and subsequently washed three times with culture medium and cultured for $24 \mathrm{~h}$ with drug-free medium (the histograms after $24 \mathrm{~h}$ from drug-free are shown as cell cycle distribution of $48 \mathrm{~h}$ ). For the concomitant treatment, the cell cycle analyses were performed after simultaneous or sequential exposures using A549 cells collected at 24 and $48 \mathrm{~h}$, respectively. Cells were treated with $0.02 \mu \mathrm{M}$ TAS-106 or $5 \mu \mathrm{M}$ CDDP, either alone or in combination.

on checkpoint-associated protein are presented in Fig. 4. The treatment of cells with TAS-106 decreased the expression of checkpoint kinase 1 (Chk1), while the expression of checkpoint kinase 2 (Chk2) apparently was unaffected. Upon incubation of the A549 cells with CDDP, a DNA damaging agent, the kinases Chk1 and Chk2 became phosphorylated at Ser-345 and Ser-19, respectively. The phosphorylation of checkpointassociated proteins causes a delay in cell cycle progression until the completion of DNA repairs. When cells were treated with a combination of TAS-106 and CDDP, the phosphorylated forms of the checkpoint-associated proteins were lower than after CDDP treatment alone. In this case, the down-regulation of the expression of the phosphorylated forms of the checkpoint-associated proteins meant that cell cycle progression occurred without sufficient repair of the DNA lesions. Therefore, these results indicated that the potentiation of the tumor growth inhibitory effect of CDDP by TAS-106 was associated with checkpoint abrogation and the subsequent increase in apoptosis induction in the tumor.

TAS-106 as cell cycle abrogator and trigger of apoptosis. We confirmed the cell cycle profiles under the conditions used in the TUNEL assay (Fig. 5). Cells treated with TAS-106 were arrested in G1 phase, while CDDP treatment resulted in Sand $\mathrm{G}_{2} \mathrm{M}$-phase arrest. On the other hand, cells treated with a combination of TAS-106 and CDDP increased the sub-G1 cell 


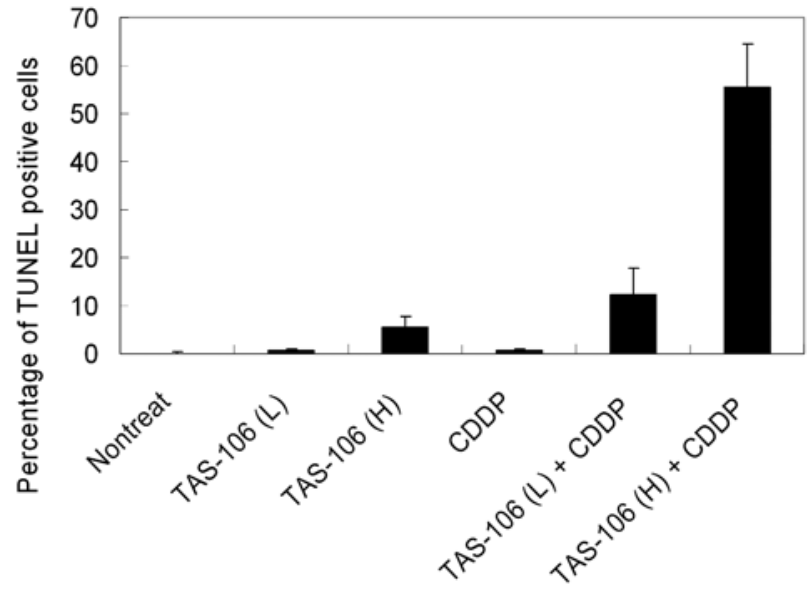

Figure 3. The percentage of TUNEL-positive cells in A549 cells treated with TAS-106, CDDP or both drugs for $72 \mathrm{~h}$. TAS-106 was set two doses $(\mathrm{L}, 0.1 \mu \mathrm{M} ; \mathrm{H}, 0.25 \mu \mathrm{M})$. CDDP was exposed at concentration of $12.5 \mu \mathrm{M}$. The percentage of TUNEL-positive cells are given as mean values \pm standard deviation (SD) of more than three independent experiments.

population in addition to a significant reduction in cells in $\mathrm{S}$ - and $\mathrm{G}_{2} \mathrm{M}$-phase, compared with the effects of CDDP monotherapy. The population in $\mathrm{S}$ - and $\mathrm{G}_{2} \mathrm{M}$-phase decreased to the level observed in control cells. In other words, cells were deprived of the protective mechanism whereby $\mathrm{G}_{2}$ blockade allows the cells to repair DNA damage and instead the cells progressed through the cell cycle.

Antitumor effect of combination therapy against subcutaneously implanted LX-1 tumors. In this experiment, we

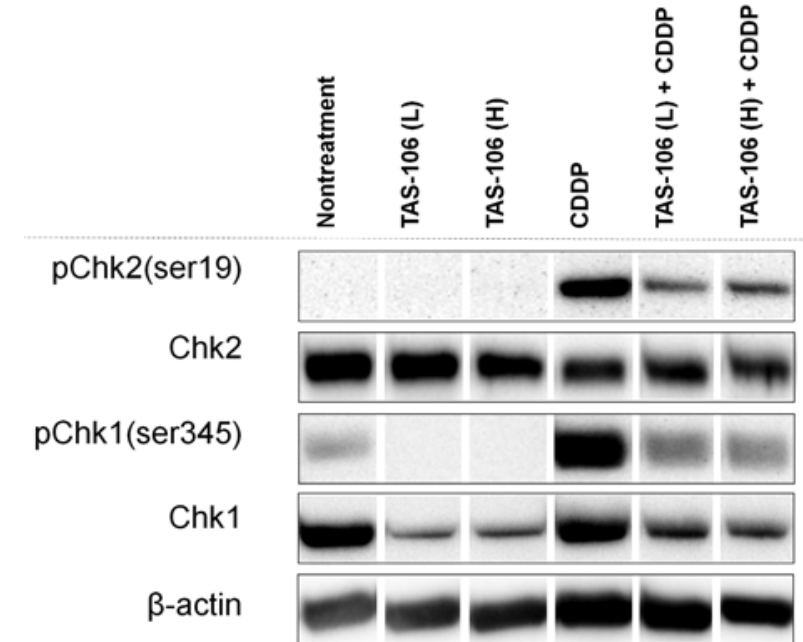

Figure 4. The expression of proteins related to the $\mathrm{G}_{2} \mathrm{M}$ checkpoint in A549 cells treated with TAS-106, CDDP or both drugs for $24 \mathrm{~h}$. TAS-106 was set two doses (L, $0.1 \mu \mathrm{M} ; \mathrm{H}, 0.25 \mu \mathrm{M})$. CDDP was exposed at concentration of $12.5 \mu \mathrm{M}$.

used non-toxic doses of TAS-106 and CDDP and evaluated the tumor growth inhibition on Day 15. Treatment with $0.3 \mathrm{mg} /$ $\mathrm{kg} /$ day TAS-106 (Days 1, 8) and $3 \mathrm{mg} / \mathrm{kg} /$ day CDDP (Day 1), administered as single agents, resulted in IR (\%) of 69.0 and $80.0 \%$, respectively. These effects were statistically significant when compared with the corresponding tumor volume in the control group ( $\mathrm{p}<0.05$, Student's t-test). On the other hand, the tumor volume after combined treatment with $0.3 \mathrm{mg} / \mathrm{kg} / \mathrm{day}$ TAS-106 and $3 \mathrm{mg} / \mathrm{kg} /$ day CDDP was dramatically reduced, with a tumor growth inhibition rate of $89.3 \%$; this value was
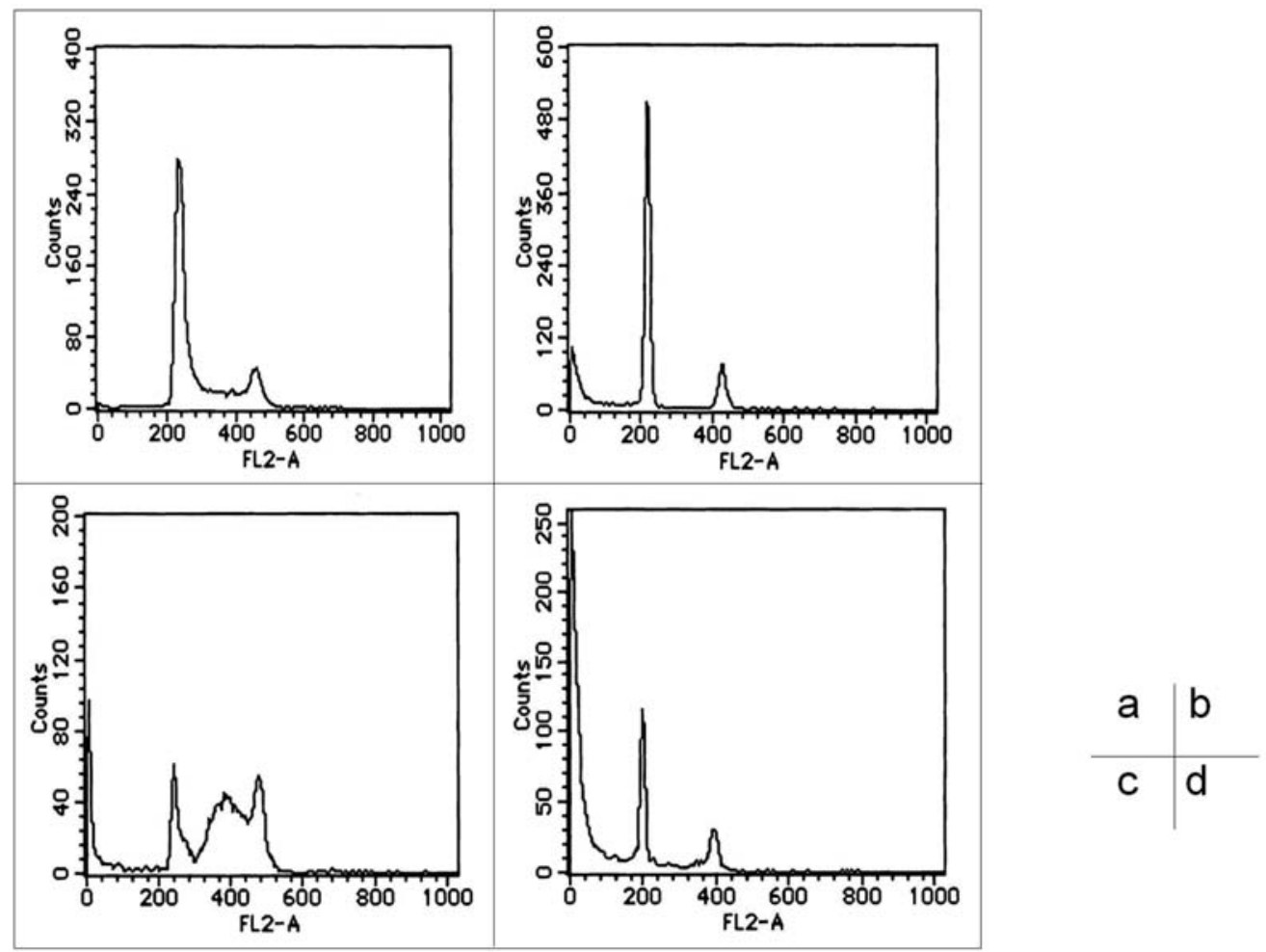

Figure 5. The cell cycle distribution under the same conditions of investigation of DNA fragmentation. The cell cycle distribution was measured by cytofluorometry in A549 cells after 72-h treatment with $0.25 \mu \mathrm{M}$ TAS-106 or $12.5 \mu \mathrm{M}$ CDDP, either alone or in combination. a, non-treatment; b, TAS-106; c, CDDP and d, combination. 

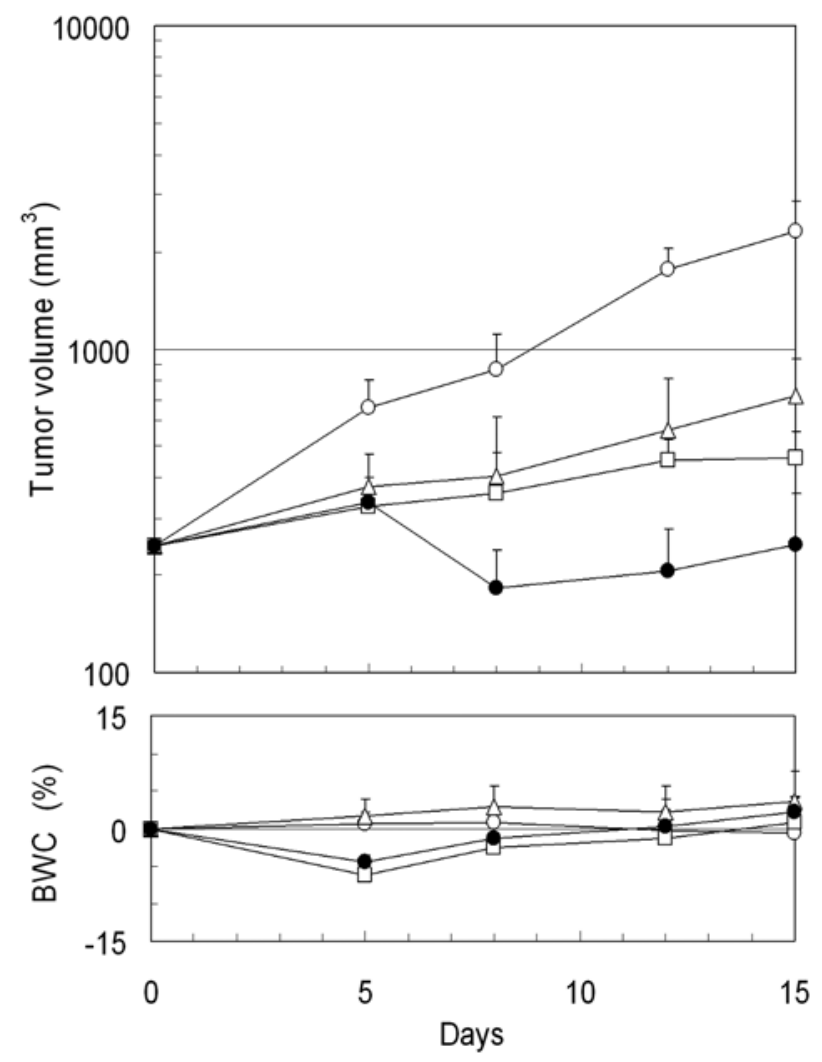

Figure 6. The combination effect of TAS-106 and CDDP was investigated in a model of human lung carcinoma, LX-1, subcutaneously transplanted into nude rat model. TAS-106 $(0.3 \mathrm{mg} / \mathrm{kg} / \mathrm{day})$ was intravenously administered on Days 1 and 8 . CDDP ( $3 \mathrm{mg} / \mathrm{kg} /$ day) was intravenously administered on Day 1. Tumor volume of control, TAS-106, CDDP and combination shows open circle, open triangle, open square and closed circle, respectively. Points, average of 6 rats (mean); bars, SD.

significantly better than those of the single drugs $(\mathrm{p}<0.01$, Student's t-test). Moreover, the combined treatment was able to induce tumor regression, a phenomenon that is strongly desired in antitumor drug evaluations (Fig. 6). The strong antitumor effects were not associated with an increase in toxicity. The time course of body weight changes in the treated animals, an indicator of drug toxicity, did not exceed a drop of $10 \%$. The hematological effects (WBC, \% Cont.) in all the groups remained at $60 \%$ or more, compared with the control values. Together, these findings suggest that the effective dose levels of both monotherapies and the combined therapy were not harmful.

Antitumor effect and life-prolonging effect of combination therapy against subcutaneously implanted OCC-1 tumors. In this experiment, we used maximum tolerated doses (MTD) of TAS-106 and CDDP and measured the tumor volume from Day 8 to Day 22. The results are shown in Fig. 7a. Tumor volume measurement was completed on Day 22, because many of the rats in the control group died before Day 22. The antitumor efficacy of the combination therapy with TAS-106 and CDDP against OCC-1 tumor xenografts was significantly enhanced, compared with that of either monotherapy $(p<0.01$, Student's t-test). Moreover, because OCC-1 tumors are suitable for evaluating antitumor efficacy using the survival time of a

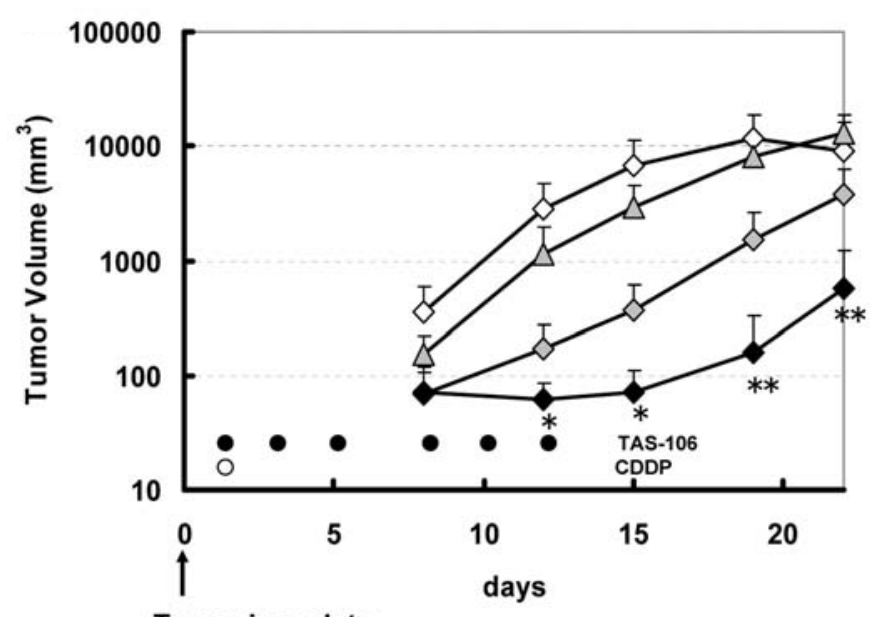

Tumor inoculate

b

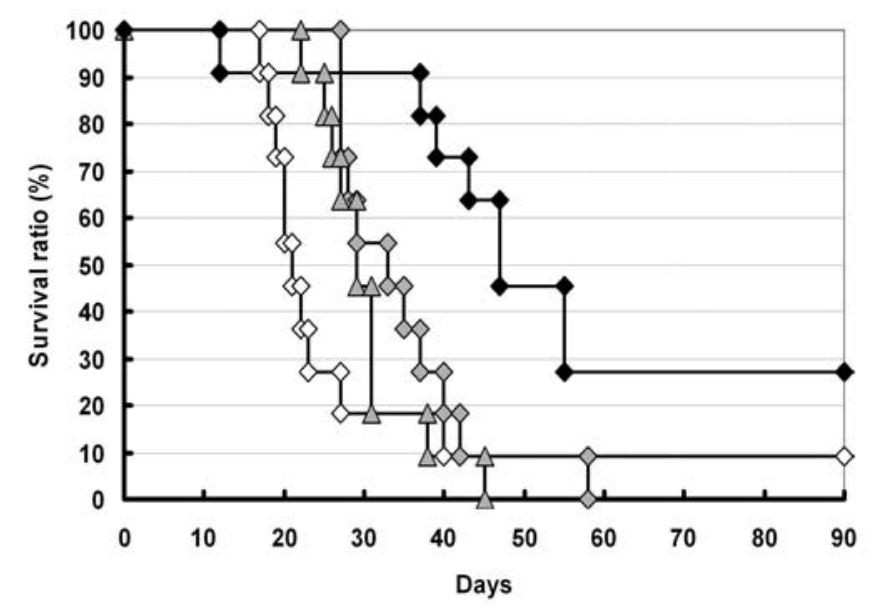

Figure 7. Antitumor effect and life-prolonging effect of combination therapy with TAS-106 and CDDP against subcutaneously implanted OCC-1 tumor. TAS-106 (0.5 mg/kg/day) were intravenously administered on Days $1,3,5$, 8,10 and 12 . CDDP $(5 \mathrm{mg} / \mathrm{kg} /$ day $)$ was intravenously administered on Day 1 . Results are representative data from one of three independent experiments. Each group consists of 11 rats. Tumor volume and survival ratio (\%) of control, TAS-106, CDDP and combination shows open diamond, shadow diamond, shadow triangle and closed diamond, respectively. (a) Tumor growth delay was confirmed by combination therapy of TAS-106 and CDDP. Points, average of 11 rats (mean); bars, SD. The antitumor efficacy for the treated with combination of TAS-106 and CDDP group was statistically significant compared with TAS-106 group and CDDP group on Days 12 to 22 ( ${ }^{*} \mathrm{p}<0.001$, ${ }^{* *} \mathrm{p}<0.0001$, Student's t-test). (b) Long-term survival of rats bearing OCC-1 tumors following treatment with combination therapy of TAS-106 and CDDP was observed. The percentage of rat viability was determined until Day 90. The survival advantage for the treated with combination of TAS-106 and CDDP group was statistically significant compared with TAS-106 group and CDDP group ( $\mathrm{p}<0.05$, Wilcoxon test).

the animals, we evaluated the life-prolonging effects. The median survival time in the control group was 21 days. The increased life-spans (ILS) values for the animal groups treated with $0.5 \mathrm{mg} / \mathrm{kg} /$ day TAS-106, $5.0 \mathrm{mg} / \mathrm{kg} / \mathrm{day}$ CDDP, or a combination of both drugs were 57.1, 38.1 and $123.8 \%$, respectively (Fig. 7b). Thus, combination therapy with 
TAS-106 and CDDP significantly prolonged the lives of the rats $(\mathrm{p}<0.05$, Wilcoxon test).

\section{Discussion}

TAS-106 has been found to exert very strong antitumor effects, prompting us to initiate its clinical evaluation. However, effective cancer treatment using single agent chemotherapy is very seldom observed; in most instances, treatments consist of a combination of several drugs. Therefore, to further explore the potential antitumor activity of a drug, positive interactions with other clinically available anticancer drugs are usually sought. As a result of such a search, synergistic cytotoxic effects were found between TAS-106 and CDDP after 72-h simultaneous exposure in A549 human lung cancer cell line (data not shown). Although the combination of TAS-106 and CDDP was found to be sufficiently effective, it remained important to establish an optimal schedule and sequence for drug exposure. Since TAS-106 cytotoxic effects are cell cycle-independent (15), it seemed reasonable to use this compound in combination with a $\operatorname{drug}(\mathrm{s})$ causing DNA damage and leading to an accumulation of cells in S- or $\mathrm{G}_{2} \mathrm{M}$-phase. On the other hand, the exposure of cancer cells to TAS-106 caused a decrease in the cell population in Sphase; therefore, combination with agents specifically affecting cells in S-phase is not advised. An example is the case of CPT-11, an S-phase-specific agent (16), whose cytotoxic effects against TAS-106 pretreated cells were reduced because of the decreased cell population in S-phase. Thus, we investigated an optimal sequence for various schedules by using 24-h treatment of each drug.

In the present study, the expression of Chk1 and Chk2 were evaluated at higher concentration than those in the investigation of the optimal exposure schedule using isobologram; the reason was to demonstrate the molecular change clearly. Such reasons may not be free of doubt that the interaction between the two drugs might have different characteristics in different concentration range. However, sequential exposure; first to CDDP and then to TAS-106 appeared to be extremely effective, and this result agrees with the present finding that TAS-106 abrogates the cell cycle checkpoints despite the presence of CDDP-induced DNA damage (Fig. 4). Thus, we concluded that TAS-106 should be applied with DNA damaging agents, either simultaneously or after pre-exposure to achieve an optional combination effect.

The arrest of the cell cycle in $\mathrm{S}$ - or $\mathrm{G}_{2} \mathrm{M}$-phase may play a protective role by allowing cells to repair DNA damage (17). The process of DNA repair is carefully controlled by a system of enzymes including Chk1, a serine/threonine kinase that is reported to be involved in S- and $\mathrm{G}_{2} \mathrm{M}$-phase arrest (18-20). In response to a variety of genotoxic stressors, such as a replicative block induced by UV light, hydroxyurea and DNA strand breaks, Chk1 is activated by phosphorylation at Ser $^{317}$ and $\operatorname{Ser}^{345}$ by an upstream kinase, ATR (21). Phosphorylated Chk1 regulates cell cycle progression through checkpoints such as the $\operatorname{cdc} 25 \mathrm{~A}$ at $\mathrm{S}$-phase and both the $\operatorname{cdc} 25 \mathrm{~A}$ and cdc25C phosphatases at $\mathrm{G}_{2} \mathrm{M}$-phase, respectively. The cdc25A and cdc25C play critical roles in mitotic entry $(22,23)$. On the other hand, another kinase, Chk2, is activated by phosphorylation at $\mathrm{Thr}^{68}$ and $\mathrm{Ser}^{19}$ by the upstream kinase
ATM, in the case of ionizing radiation-induced DNA damage (24).

In the present study, we confirmed that TAS-106 decreased the expression level of Chk1. As reported by Shannon and Greg (25), Chk1 interference RNA combined with the partial inhibition of DNA replication was sufficient to evoke a response to DNA damage. Both cyclin-dependent kinases $(\mathrm{Cdk})$ and Chk1 inhibitors enhanced the cytotoxicity of etoposide, a DNA-damaging agent. Similar effects were reported by Bartz et al (26), where the silencing of checkpoint genes, ATR and Chk1, also enhanced sensitivity to cisplatin. These studies indicate that the down-regulation of the Chk1 expression level is one of the important factors for antitumor efficacy. Although the main mechanism of TAS-106 involves the inhibition of RNA synthesis, its additional ability to abrogate a checkpoint of the cell cycle seems to be an important feature, particularly for combined treatment. Many $\mathrm{G}_{2}$ checkpoint abrogators have already been reported in the case of anticancer drugs (27). In fact, Chk1, Chk2, ATM, ATR and PP2A belong to this type of target, and Chk1, in particular, is targeted by many anticancer drugs: staurosporin (28), UCN-01 (28,29), Go6976 (30) and SB-218078 (28), to name a few. Moreover, the dysregulation of cell cycle checkpoints is now recognized as a salient feature of the malignant transformation process. Some studies have suggested the involvement of Chk1 ablation in oncogenesis and/or advancing tumor grade $(31,32)$.

The careful analysis of the cell cycle profiles, performed under the conditions used in the TUNEL assay, indicated the presence of S- and $\mathrm{G}_{2} \mathrm{M}$-phase arrests and $\mathrm{G} 1$ block induced by CDDP and TAS-106, respectively (Fig. 5). The treatment of the cells simultaneously with CDDP and TAS-106, on the other hand, resulted in the significant reduction of the cell population in $\mathrm{S}$ - and $\mathrm{G}_{2} \mathrm{M}$-phase induced by CDDP alone, in consequence leading to an increase of sub-G1 phase population. Probably, the cells damaged by CDDP were able to progress into next phase due to the lack of cell cycle checkpoints, however most of them entered the apoptotic stage because of the DNA damaging effect of CDDP. Moreover, when we observed morphology, the acridine orange/ethidium bromide staining of the cells revealed no remarkable change in TAS-106-treated cells, however, cell swelling was observed in CDDP-treated cells without obvious apoptotic events (data not shown). The combined treatment resulted, however, in very dramatic induction of apoptosis. At this stage, it is difficult to clarify the exact mechanism of this important phenomenon. These promising in vitro results prompted us to initiate in vivo evaluation of these interesting properties.

The OCC-1 tumors grew by 20 -fold in one week; in other words, the proliferation of this tumor was very rapid. So, in the study using OCC-1 tumors, TAS-106 was administered three times a week using MTD for this schedule. The OCC-1 cancer responded poorly to CDDP treatment, a golden standard for that type of tumor. However, combined treatment with CDDP and TAS-106 significantly increased the antitumor effect, both in terms of tumor size reduction (Fig. 7a) and the prolongation of the life-spans of the treated animals (Fig. 7b). Moreover, 5-FU, a key drug for head and neck cancer, was not effective against OCC-1 tumors (data not shown), while TAS-106, even administered alone, exerted a strong effect. 
Thus, TAS-106 may be an effective therapeutic against head and neck cancers that are refractory to CDDP and 5-FU. A similar strong antitumor activity was demonstrated in the case of LX-1 cancer, where significant antitumor activity was even associated with tumor regression (Fig. 6). The dosage of TAS-106 used for the study on LX-1 was $1 / 20$ of MTD, so that the efficacy of the drug at low dose exposures could be confirmed. Despite this low dose of TAS-106, which produced no significant toxic manifestations, TAS-106 enhanced the antitumor activity of CDDP, resulting in tumor regression.

In conclusion, we have shown that TAS-106 was able to potentiate the effects of CDDP, both in vitro and in vivo. Furthermore, the rationale for this drug combination is based not only on a difference in the main mechanisms of action of TAS-106 and CDDP, but also on the additional ability of TAS-106 to abrogate the cell cycle checkpoints, thus preventing the repair of damaged DNA. These results show that TAS-106 is a potential candidate drug for combination therapy with CDDP.

\section{References}

1. Matsuda A and Sasaki T: Antitumor activity of sugar-modified cytosine nucleosides. Cancer Sci 95: 105-111, 2004.

2. Hattori H, Tanaka M, Fukushima M, Sasaki T and Matsuda A: Nucleosides and Nucleotides. 158. 1-(3-C-Ethynyl- $\beta$-D-ribopentofuranosyl) cytosine, 1-(3-C-Ethynyl-ß-D-ribo-pentofuranosyl) uracil, and their nucleobase analogues as new potential multifunctional antitumor nucleosides with a broad spectrum of activity. J Med Chem 39: 5005-5011, 1996

3. Tabata S, Tanaka M, Matsuda A, Fukushima M and Sasaki T: Antitumor effect of a novel multifunctional antitumor nucleoside, 3'-ethynylcytidine, on human cancers. Oncol Rep 3: 1029-1034, 1996.

4. Shimamoto Y, Fujioka A, Kazuno H, Murakami Y, Ohshimo H, Kato T, Matsuda A, Sasaki T and Fukushima M: Antitumor activity and pharmacokinetics of TAS-106, 1-(3-C-Ethynyl- $\beta$ D-ribo-pentofuranosyl) cytosine. Jpn J Cancer Res 92: 343-351, 2001.

5. Tabata S, Tanaka M, Endo Y, Obata T, Matsuda A and Sasaki T: Anti-tumor mechanisms of 3'-ethynyluridine and ethynylcytidine as RNA synthesis inhibitors: development and characterization of 3'-ethynyluridine resistant cells. Cancer Lett 116: 225-231, 1997.

6. Takatori S, Kanda H, Takenaka K, Wataya Y, Matsuda A, Fukushima M, Tanaka M and Sasaki T: Antitumor mechanisms and metabolism of the novel antitumor nucleoside analogues, $1-$ (3-C-Ethynyl-B-D-ribo-pentofuranosyl) cytosine and 1-(3-CEthynyl-B-D-ribo-pentofuranosyl) uracil. Cancer Chemother Pharmacol 44: 97-104, 1999.

7. Koizumi K, Shimamoto Y, Azuma A, Wataya Y, Matsuda A, Sasaki T and Fukushima M: Cloning and expression of uridine/ cytidine kinase cDNA from human fibrosarcoma cells. Int J Mol Med 8: 273-278, 2001

8. Naito T, Yokogawa T, Takatori S, Goda K, Hiramoto A, Sato A, Kitade Y, Sasaki T, Matsuda A, Fukushima M, Wataya Y and Kim HS: Role of RNase L in apoptosis induced by 1-(3-CEthynyl-3-D-ribo-pentofuranosyl) cytosine. Cancer Chemother Pharmacol (In press).

9. Sorenson CM and Eastman A: Influence of cis-diamminedichloroplatinum(II) on DNA synthesis and cell cycle progression in excision repair proficient and deficient chinese hamster ovary cells. Cancer Res 48: 6703-6707, 1988.

10. Sorenson CM, Barry MA and Eastman A: Analysis of events associated with cell cycle arrest at $\mathrm{G}_{2}$ phase and cell death induced by cisplatin. J Natl Cancer Inst 82: 749-755, 1990.

11. Carmichael J, DeGraff WG, Gazdar AF, MinnaJD and Mitchell JB: Evaluation of a tetrazolium-based semiautomated colorimetric assay: assessment of chemosensitivity testing. Cancer Res 47: 936-942, 1987.

12. Steel GG and Peckham MJ: Exploitable mechanisms in combined radiotherapy - chemotherapy: the concept of additivity. Int J Radiat Oncol Biol Phys 5: 85-93, 1979.
13. Tsai CM, Gazdar AF, Venzon DJ, Steinberg SM, Dedrick RL, Mulshine JL and Kramer BS: Lack of in vitro synergy between etoposide and cis-diamminedichloroplatinum(II). Cancer Res 49: 2390-2397, 1989.

14. Li X, Traganos F, Melamed MR and Darzynkiewicz Z: Single step procedure for labeling DNA strand breaks with fluorescein- or BODIPY-conjugated deoxynucleotides. Detection of apoptosis and bromodeoxyuridine incorporation. Cytometry 20: 172-180, 1995.

15. Kazuno H, Shimamoto Y, Tsujimoto H, Fukushima M, Matsuda A and Sasaki T: Mechanism of action of a new antitumor ribo-nucleoside, 1-(3-C-ethynyl-B-D-ribo-pentofuranosyl) cytosine (ECyd, TAS-106), differs from that of 5-fluorouracil. Oncol Rep 17: 1453-1460, 2007.

16. Aoyagi Y, Kobunai T, Utsugi T, Oh-hara T and Yamada Y: In vitro antitumor activity of TAS-103, a novel quinoline derivative that targets topoisomerase I and II. Jpn J Cancer Res 90: 578-587, 1999.

17. Cliby WA, Lewis KA, Lilly KK and Kaufmann SH: S phase and $\mathrm{G}_{2}$ arrests induced by Topoisomerase I poisons are dependent on ATR kinase function. J Biol Chem 277: 1599-1606, 2002.

18. Bartek $\mathrm{J}$ and Lukas J: Chk1 and Chk2 kinases in checkpoint control and cancer. Cancer Cell 3: 421-429, 2003.

19. Zhou BB and Bartek J: Targeting the checkpoint kinases: chemosensitization versus chemoprotection. Nat Rev Cancer 4: 216-225, 2004

20. Xiao Z, Chen Z, Gunasekera AH, Sowin TJ, Rosenberg SH, Fesik $\mathrm{S}$ and Zhang $\mathrm{H}$ : Chk1 mediates $\mathrm{S}$ and $\mathrm{G}_{2}$ arrests through cdc25A degradation in response to DNA-damaging agents. J Biol Chem 278: 21767-21773, 2003.

21. Zhao H and Piwnica-Worms H: ATR-mediated checkpoint pathways regulate phosphorylation and activation of human Chk1. Mol Cell Biol 21: 4129-4139, 2001.

22. Peng CY, Graves PR, Thoma RS, Wu Z, Shaw AS and Piwnica-Worms H: Mitotic and G2 check point control: regulation of 14-3-3 protein binding by phosphorylation of Cdc25C on serine-216. Science 277: 1501-1505, 1997.

23. Ferguson AM, White LS, Donovan PJ and Piwnica-Worms H: Normal cell cycle and checkpoint responses in mice and cells lacking Cdc25B and Cdc25C protein phosphatases. Mol Cell Biol 25: 2853-2860, 2005.

24. Michael BK: Cell cycle: Checking two steps. Nature 410: 766-767, 2001.

25. Shannon LM and Greg HE: Cdk inhibition in human cells compromises Chk1 function and activates a DNA damage response. Cancer Res 65: 780-786, 2005.

26. Bartz SR, Zhang Z, Burchard J, Imakura M, Martin M, Palmieri A, Needham R, Guo J, Gordon M, Chung N, Warrener P, Jackson AL, Carleton M, Oatley M, Locco L, Santini F, Smith T, Kunapuli P, Ferrer M, Strulovici B, Friend SH and Linsley PS: Small interfering RNA screens reveal enhanced cisplatin cytotoxicity in tumor cells having both BRCA network and TP53 disruptions. Mol Cell Biol 26: 9377-9386, 2006.

27. Kawabe $\mathrm{T}: \mathrm{G}_{2}$ checkpoint abrogators as anticancer drugs. Mol Cancer Ther 3: 513-519, 2004.

28. Jackson JR, Gilmartin A, Imburgia C, Winkler JD, Marshall LA and Roshak A: An indolocarbazole inhibitor of human checkpoint kinase (Chk1) abrogates cell cycle arrest caused by DNA damage. Cancer Res 60: 566-572, 2000.

29. Graves PR, Yu L, Schwarz JK, Gales J, Sausville EA, O'Connor PM and Worms HP: The Chk1 protein kinase and the $\mathrm{Cdc} 25 \mathrm{C}$ regulatory pathways are targets of the anticancer agent UCN-01. J Biol Chem 275: 5600-5605, 2000.

30. Kohn EA, Yoo CJ and Eastman A: The protein kinase C inhibitor Go6976 is a potent inhibitor of DNA damage-induced S and G2 cell cycle checkpoints. Cancer Res 63: 31-35, 2003.

31. Menoyo A, Alazzouzi H, Espin E, Armengol M, Yamamoto H and Schwartz S Jr: Somatic mutations in the DNA damageresponse genes ATR and Chk1 in sporadic stomach tumors with microsatellite instability. Cancer Res 61: 7727-7730, 2001.

32. Vassileva V, Millar A, Briollais L, Chapman W and Bapat B: Genes involved in DNA repair are mutational targets in endometrial cancers with microsatellite instability. Cancer Res 62: 4095-4099, 2002. 